\title{
Congenital syphilis in aborted second trimester fetus: diagnosis by histological study
}

\author{
HERBERT BRAUNSTEIN \\ From the Department of Laboratories, San Bernardino County Medical Center, \\ San Bernardino, California 92404, USA
}

SUMMARY A case of congenital syphilis in an aborted fetus in the second trimester of pregnancy was documented by demonstration of spirochaetes in the fetal liver, despite the fact that diagnostic studies had not been performed on the mother. Suspicion was aroused by the gross and microscopic appearance of the placenta, which appeared identical with that described in proven cases of congenital syphilis. Although these changes have been alleged to be non-specific, the rarity with which such well-developed lesions are encountered in placentas in other circumstances dictates the necessity for further study when they are observed.

Recently we observed an example of congenital syphilis in which abnormalities in the placenta of a macerated fetus, spontaneously aborted in an emergency room in the second trimester of pregnancy, prompted study of the fetus for spirochaetes. The diagnosis of congenital syphilis was established by the demonstration of countless spirochaetes in the autolysed fetal liver.

\section{Case report}

A 19-year-old Caucasian woman was admitted to the emergency room with an impending miscarriage. Her past history revealed that the last menstrual period had occurred approximately four and a half months previously with unprotected coitus thereafter. Two days before admission bleeding had occurred, followed the next day by cramping pain. The patient was a transient, allegedly affianced to the father of the fetus, and travelling through the area. A history of promiscuity was not obtained, and the pregnancy was alleged to be her first.

Physical examination revealed a pale young woman with numerous 'condylomata acuminata' and much yellowish vaginal discharge. In the vagina the products of conception could be seen bulging from the dilated cervical os. Haemoglobin was $9.6 \mathrm{~g} / \mathrm{dl}$, blood type $\mathrm{O}, \mathrm{Rh}$ positive, blood sugar $91 \mathrm{mg} / \mathrm{dl}$. No test for syphilis was performed.

A spontaneous, apparently complete abortion occurred. The patient was managed as an outpatient and discharged after about three hours of observation. The fetus measured $128 \mathrm{~mm}$ in crownrump length. The placenta was pale and extremely firm, measuring $7.5 \mathrm{~cm}$ in diameter and about $1 \mathrm{~cm}$ in thickness. The fetus manifested maceration, and gross interpretation of organ changes was not possible.

Microscopically the placenta was well preserved, with marked enlargement of the placenta villi, which showed both perivascular fibroblastic and endothelial proliferation. The small vessels were quite bloodless. The surface epithelium was a syncytial monolayer (Fig. 1). Rare mononuclear inflammatory cells were noted. Because of the gross and microscopic appearance of the placenta, additional sections were obtained and stained.

Levaditi stain of the placenta did not reveal unequivocal spirochaetes, although two or three suspicious structures were noted. Haematoxylin and eosin stained sections of the fetal liver showed autolytic change and haemopoiesis. Levaditi stains of the liver revealed countless spirochaetes (Fig. 2). $X$-ray studies and sections of the long bones did not reveal evidence of osteochondritis. Levaditi stains of the lung, heart, and spleen showed occasional spirochaetes in the lung, but none in the heart or spleen.

\section{Discussion}

The relative paucity of spirochaetes in the placenta 


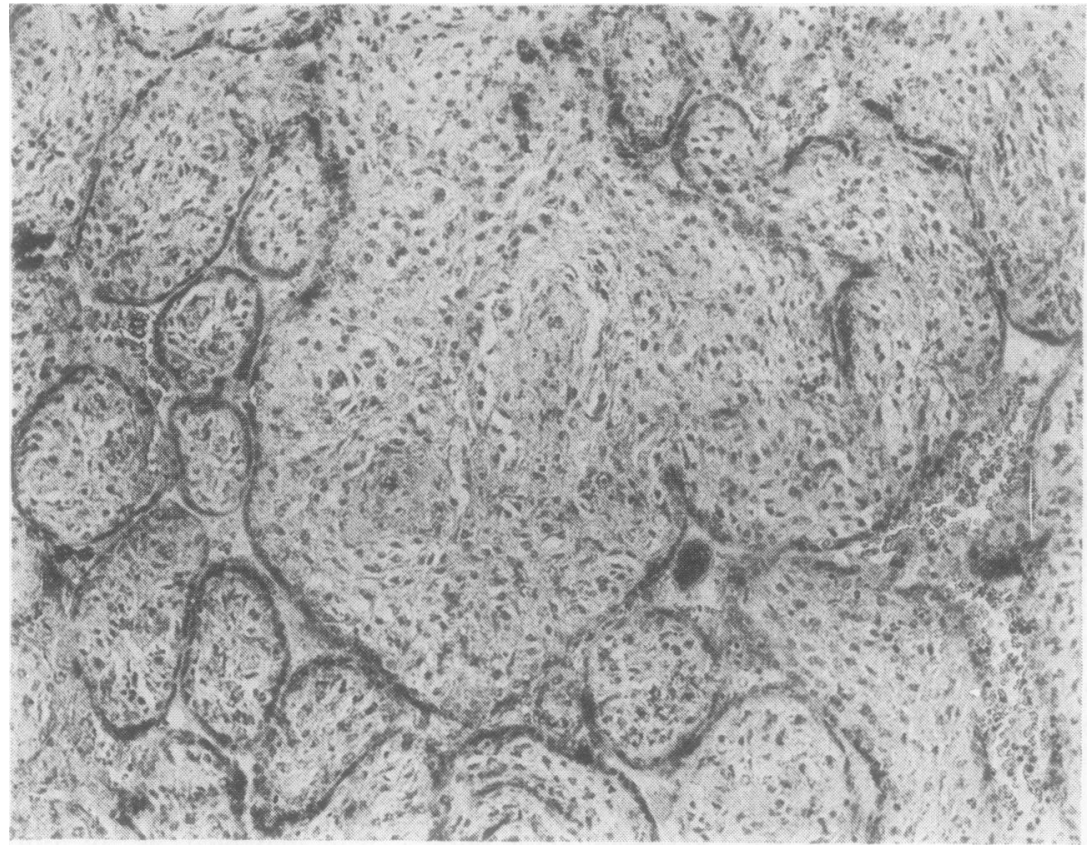

Fig. 1 A representative section from the placenta of the case reported shows marked enlargement and cellularity of villi, crowding and reduction of intervillous spaces, and almost complete absence of blood in villi reflecting endothelial swelling and proliferation and perivascular fibroblastic proliferation. (Haematoxylin and eosin $\times 100$ )

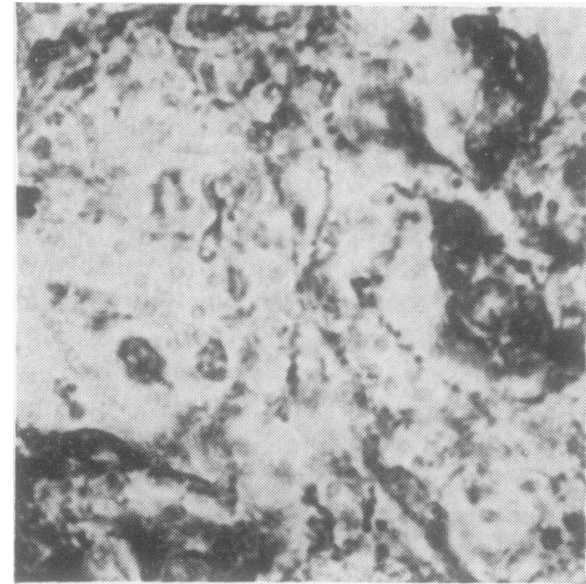

Fig. 2 Oil immersion photomicrograph of the liver of the aborted fetus shows numerous black staining spirochaetes, both intact and fragmented. (Levaditi $\times 960)$

in congenital syphilis (McCord, 1934) and their abundance in the liver (Von Haam, 1971) have been previously noted. The latter has been attributed to the 'incubator' effect of the uterus in abortion or stillbirth, the spirochaetes multiplying rapidly in the autolysed liver within the retained fetus (Stokes, 1936).

Abnormalities in the placenta in maternal syphilis are uncommon, but they have been described with

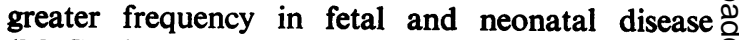
(McCord, 1934). McCord listed the features of the $\stackrel{\mathbb{Q}}{\stackrel{2}{2}}$ syphilitic placenta as diffuse enlargement and $\overrightarrow{\vec{P}}$ crowding of villi; presence of connective tissue in 3 varying density; increase in stromal cells; and lessening or absence of villous blood vessels. In subsequent studies the specificity of these changes has been challenged, and it has been contended that ? similar alterations may be encountered in the placenta in many other circumstances (Montgomery, 1936; Whipple and Dunham, 1938; Benirschke and Driscoll, 1967).

Russell and Altshuler (1974) more recently des- 옥 cribed the morphological abnormalities of the $\frac{D}{0}$ placenta at term in three proven cases of congenital syphilis. These included: relative immaturity of $N$ villi; focal (largely decidual) plasma cell infiltrations; $N$ and vascular obliteration due to endothelial and $\mathcal{N}$ perivascular fibroblastic proliferation. In one in- $\omega$ stance, there were granulomas. Neither the first nor the second change appears to be specific for syphilis, and the first would be of major significance only at $\Phi$ term. The case here described manifested only the third feature, which appears to be the most specific manifestation of postnatal syphilis as well (Von Haam, 1971).

The frequency with which 'subacute and chronic $\stackrel{D}{\triangle}$ inflammation' is seen in non-syphilitic placentas, studied histologically, is estimated at $5 \%$ by 
Benirschke and Driscoll (1967). The same authors state that occasional placentas show obliterative vasculitis without syphilis, but they do not illustrate such a lesion. Their photomicrograph of a syphilitic placenta appears identical with ours. While obliterative vasculitis has been described in ischaemia, including prolonged placental retention, the characteristic fibromuscular sclerosis of stem arteries is predominantly an endovascular and not a perivascular process (Fox, 1975). Furthermore, in contrast to the predominantly proliferative process of congenital syphilis, with villous enlargement and crowding, retention is characterised by cellular degeneration, atrophy, and disappearance of blood vessels, as well as fibrosis with shrinkage of villi (Benirschke and Driscoll, 1967; Fox, 1975).

At San Bernardino County Medical Center, placentas from more than 50 fetuses of similar gestational age (crown-rump length $120-170 \mathrm{~mm}$ ) have been examined in the surgical pathology laboratory each year for the last three years. The case described represents the only instance in which the appearance of the placenta prompted an investigation for spirochaetes. Review of the sections of placenta from the $\mathbf{4 0}$ most recent similar fetuses personally studied by the author did not reveal any similar histological changes. Minor degrees of oedema and fibrosis of placental villi were observed in a few, but no proliferative vasculitis.

Perhaps the successful documentation of congenital syphilis in this instance was serendipitic, but it appears at least equally probable that the placental changes, if not diagnostic, are character- istic. It is recommended that further studies be initiated whenever similar findings are encountered (Benirschke and Driscoll, 1967).

\section{References}

Benirschke, K., and Driscoll, S. G. (1967). The Pathology of the Human Placenta. p. 512. Springer, New York.

Fox, H. (1975). Morphological pathology of the placenta. In The Placenta and its Maternal Supply Line, edited by P. Gruenwald, chapter 2, p. 366. University Park Press, Baltimore.

McCord, J. R. (1934). Syphilis of the placenta. The histologic examination of 1085 placentas of mothers with strongly positive blood Wasserman reactions. American Journal of Obstetrics and Gynecology, 28, 743-750.

Montgomery, T. L. (1936). Fibrosis of the placenta: its significance in the normal and in the syphilitic organ. American Journal of Obstetrics and Gynecology, 31, 253-267.

Russell, P., and Altshuler, G. (1974). Placental abnormalities of congenital syphilis: a neglected aid to diagnosis. American Journal of Diseases of Children, 128, 160-163.

Stokes, J. H. (1936). Modern Clinical Syphilology, 2nd edition, p. 1400. W. B. Saunders, Philadelphia. Cited by Whipple and Dunham (1938).

Von Haam, E. (1971). Venereal diseases and spirochetal infections. Chap 10. In Pathology, 6th edition, edited by W. A. D. Anderson, Vol. 1, pp. 341-364. G. V. Mosby, St. Louis.

Whipple, D. V., and Dunham, E. C. (1938). Congenital syphilis. Part 1. Incidence, transmission, diagnosis. Journal of Pediatrics, 12, 386-398. 